\title{
The impact of COVID-19 on rural population: $A$ retrospective study
}

\author{
๑İbrahim Halil İnanç ${ }^{1}$, \Nurbanu Bursa ${ }^{2}$, \Ahmet Gültepe ${ }^{3}$, \Cengiz Şabanoğlu ${ }^{4}$ \\ ${ }^{1}$ Besni State Hospital, Department of Cardiology, Adıyaman, Turkey \\ ${ }^{2}$ Hacettepe University, Department of Statistics, Faculty of Science, Ankara, Turkey \\ ${ }^{3}$ Gölbaşı State Hospital, Department of Internal Diseases, Adıyaman, Turkey \\ ${ }^{4}$ Kırıkkale Yüksek İhtisas Hospital, Department of Cardiology, Kırıkkale, Turkey
}

Cite this article as: İnanç İH, Bursa N, Gültepe A, Şabanoğlu C. The impact of COVID-19 on rural population: A retrospective study. J Health Sci Med 2021; 4(5): 722-727.

\begin{abstract}
Objective: The COVID-19 outbreak became a major global health concern. There are some differences between urban and rural areas that may determine the impact of a viral pandemic. In our study, we aimed to investigate and present epidemiological, demographic, clinical, and radiological data relating to a rural area.

Material and Method: This retrospective two-center study involved 2234 patients tested for COVID-19 between March 30 th and July 15th, 2020. All patients were analysed for clinical, demographic, and radiological data.

Results: Of the patients; 1309 (58.6\%) were male, 925 (41.4\%) female and 259 (11\%) were PCR positive, and 1975 (89\%) PCR negative. Of the PCR positive group, 121 (46.7\%) were male and 138 (53.3\%) female. The mean age was $38 \pm 18.5$. Twenty-nine (11.2\%) patients needed intensive care support. Twelve (4.6\%) patients died due to COVID-19: two of them due to COVID19-related myocardial infarction and ten of them due to severe pneumonia, acute respiratory distress syndrome (ARDS), multiorgan dysfunction, or septic shock. The case fatality rate (CFR) was $4.6 \%$.

Conclusion: Age, sex, hypertension, diabetes, asthma, and heart failure were associated with COVID-19 infection. The risk of infection was higher in patients older than 20 years $(\mathrm{p}<0.001)$ and females (OR: 1.636; $\mathrm{p}<0.001)$. Patients with hypertension (OR: 2.281; $\mathrm{p}<0.001)$, diabetes (OR: 1.013; $\mathrm{p}=0.039)$, asthma (OR: $2.786 ; \mathrm{p}=0.001)$ or heart failure $(\mathrm{OR}: 2.610 ; \mathrm{p}=0.006) \mathrm{had}$ a significantly higher risk of infection.
\end{abstract}

Keywords: COVID-19, Viral pandemic, Rural area

\section{INTRODUCTION}

In December 2019, a cluster of unidentified pneumonia cases appeared in Wuhan, China. The National Health Commission (NHC) of the People's Republic of China later announced that a novel coronavirus was responsible for the outbreak (1). The virus is genetically similar to bat coronaviruses and shares about $79 \%$ and $50 \%$ of its genetic sequence with the coronaviruses that are responsible for the Middle East respiratory syndrome (MERS) and severe acute respiratory syndrome (SARS), respectively (2). The virus, named the 2019 novel coronavirus (2019-nCoV), has rapidly spread across China and other countries and has become a major global health concern. On February 11th 2020, the World Health Organization (WHO) announced a name for the epidemic disease caused by 2019-nCoV: coronavirus disease (COVID-19) (3). Evidence of human-to-human transmission through respiratory droplets, contact and, even fecal-oral transmission was found (4). Because of its high transmissibility and its incubation period, the number of cases worldwide has reached approximately 11 million, with 404,396 deaths (5).

While some infected patients present with symptoms of mild respiratory tract infection, some develop severe pneumonia, ARDS, or multiple organ failure, leading to death (5).

According to a recent analysis, male patients have a markedly increased risk of developing a severe disease compared with females (6). Smoking and the presence of concomitant diseases such as hypertension, diabetes, cardiovascular disease, and respiratory disease are factors significantly associated with a severe prognosis (7). 
Although clinical features of COVID-19 include a dry cough, fever, diarrhea, vomiting, and myalgia, some patients with multiple comorbidities present with severe infection complications such as acute kidney injury (AKI) and symptoms of ARDS. Currently, there are few studies that define the pathophysiological characteristics of COVID-19, and there is an uncertainty about its mechanism of spread. Basic hand hygiene, avoiding travel to high-risk areas, and avoiding contact with symptomatic individuals are all important in preventing the disease despite on going vaccine development for COVID-19 $(8,9)$.

Since rural regions have distinct demographic and health infrastructure issues requiring tailored approaches to service delivery, there are few studies about the effects of the COVID-19 pandemic on rural populations. Adiyaman is a province in south-central Turkey that has an area of 7,606.16 $\mathrm{km}^{2}$ and a population of 626,465 as of 2019 (Figure 1). In our study, we aimed to present clinical and demographic data relating to patients from two neighboring rural areas in Turkey.

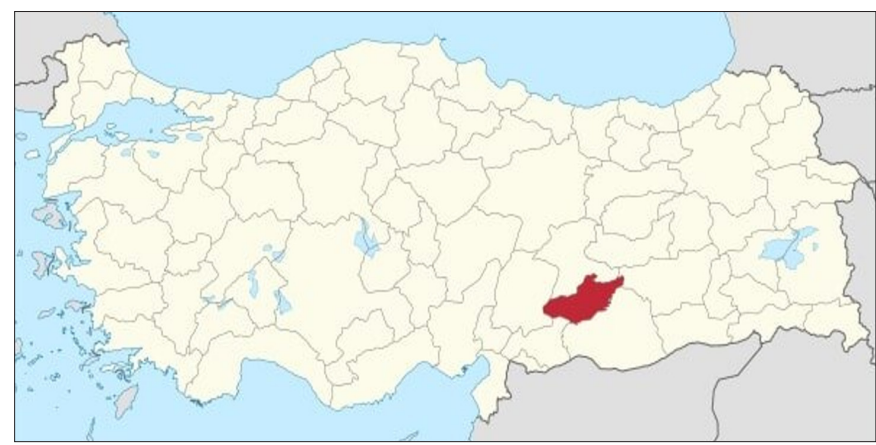

Figure 1. Turkey map showing the location of Adiyaman province

\section{MATERIAL AND METHOD}

\section{Study Design and Clinical Parameters}

The study included 2234 patients from two centers in Adiyaman, Turkey between March $30^{\text {th }}$ and July $15^{\text {th }}$, 2020. Clinical, medical history, and radiological data were obtained through the electronic patient database retrospectively. Patients who had signs and symptoms of acute respiratory disease or clinical symptoms that could not be explained by any other cause/disease, individuals with or without symptoms who had spent more than 15 minutes with or had been less than one meter apart from COVID-19-positive patients, individuals sharing the same home or office with infection-suspected patients, those who recently traveled abroad, and babies born from suspected COVID-19-positive mothers were included. Individuals who had not been in contact with COVID-19-positive patients, had no recent history of travel abroad, and had no complaints were excluded. A combination of nasopharyngeal and oropharyngeal swabs were taken. Reverse transcription-polymerase chain reaction (RT-PCR) results were considered the reference standard. The swab test was repeated at least one day later in patients who had typical symptoms and positive CT findings but negative test results. The formula below was used to measure the case fatality rate (CFR):

\section{CFR (\%) $=($ Number of deaths due to COVID-19/ Number of closed cases of COVID-19) $\times 100$.}

Ethics This study was approved by Clinical Research Ethics Committee of Adryaman University (Date 21.07.2020, Decision No: 2020/7-32). All procedures were carried out in accordance with the ethical rules and the principles of the Declaration of Helsinki.

\section{Imaging Interpretation}

Chest X-ray (CXR) was the first-line imaging modality used for patients with suspected COVID-19. Computed tomography $(\mathrm{CT})$ was performed in patients with positive $\mathrm{X}$-ray findings, such as the presence of bilateral nodular/ peripheral ground-glass opacities and consolidation. The CT and CXR images of the relevant patients were reviewed by two thoracic radiologists who were not privy the patients' test results. Ground-glass opacities (GGO), a crazy-paving pattern appearance, vascular dilatation in the area of GGO, air space consolidation, bronchovascular thickening in the lesion, and traction bronchiectasis were reported as positive CT findings. The CO-RADS classification system was used to assess the pulmonary involvement of COVID-19. According to this scoring system, numbers ranging from one to five $(1 .<5 \%$ involvement; $2.5 \%-25 \%$ involvement; 3 . $26 \%-49 \%$ involvement; $4.50 \%-75 \%$ involvement; 5 . > $75 \%$ involvement) are used and reflect the severity of the disease.

\section{Statistical Analysis}

Statistical analyses were applied using SPSS 23 for Windows (SPSS Inc., Chicago, IL, U.SA.). The distribution of the variables was examined using the Kolmogorov-Smirnov test. The continuous variables, depending on whether they had normal distribution or not, were presented as mean \pm standard deviation or median \pm interquartile range, respectively. The categorical variables were displayed as percentages.

For the comparison between the PCR positive group and the PCR negative group, the Mann-Whitney U test and the Chi-squared test were appropriately used. Additionally, after the Chi-squared test for age groups was applied, post-hoc testing with the Bonferroni adjustment was carried out to determine the age group that was significant (10).

Binomial logistic regression analysis was also performed to evaluate the impact of variables found significant in Table 4 on the odds ratio for the eventuality of COVID- 
19-positive cases. In all analyses (except the age group variables in Table 1, where because of the Bonferroni adjustment, the p-value was accepted as 0.006 for these variables), two-tailed $\mathrm{p}<0.05$ was accepted as statistically significant.

\begin{tabular}{|c|c|c|c|}
\hline & $\begin{array}{l}\operatorname{PCR}(+) \\
(n=259)\end{array}$ & $\begin{array}{c}\text { PCR (-) } \\
(n=1975)\end{array}$ & $p$ value \\
\hline Age (years) & $38 \pm 18.5$ & $33 \pm 11$ & $0.030^{*}$ \\
\hline Age <6 (n, \%) & $11(4.2 \%)$ & $57(2.9 \%)$ & 0.230 \\
\hline Age 6-20 (n, \%) & $48(18.5 \%)$ & $270(13.7 \%)$ & 0.035 \\
\hline Age $21-50(n, \%)$ & $117(45.2 \%)$ & $1248(63.2 \%)$ & $<0.001^{\star}$ \\
\hline Age > $50(\mathrm{n}, \%)$ & $83(32 \%)$ & $400(20.3 \%)$ & $<0.001^{\star}$ \\
\hline $\operatorname{Sex}(n, \%)$ & & & $<0.001^{\star}$ \\
\hline Male & $121(46.7 \%)$ & $1188(60.1 \%)$ & $<0.001^{\star}$ \\
\hline Female & $138(53.3 \%)$ & $787(39.9 \%)$ & $<0.001^{*}$ \\
\hline Hypertension (n, \%) & $56(21.6 \%)$ & $197(10 \%)$ & $<0.001^{\star}$ \\
\hline $\mathrm{DM}(\mathrm{n}, \%)$ & $27(10.4 \%)$ & $77(3.9 \%)$ & $<0.001^{\star}$ \\
\hline Smoking (n, \%) & $74(28.6 \%)$ & $624(31.6 \%)$ & 0.354 \\
\hline COPD (n, \%) & $15(5.8 \%)$ & $66(3.3 \%)$ & 0.162 \\
\hline Asthma (n, \%) & $16(6.2 \%)$ & $39(2 \%)$ & $0.001^{*}$ \\
\hline Heart Failure (n, \%) & $19(7.3 \%)$ & $35(1.8 \%)$ & $<0.001^{\star}$ \\
\hline Symptomatic (n, \%) & $148(57.1 \%)$ & $414(21 \%)$ & $<0.001^{\star}$ \\
\hline Fever & $66(44.3 \%)$ & $97(4.9 \%)$ & $<0.001^{\rtimes}$ \\
\hline Cough & $140(93.3 \%)$ & $364(18.4 \%)$ & 0.087 \\
\hline Shortness of breath & $62(41.6 \%)$ & $198(10 \%)$ & 0.213 \\
\hline Tiredness & $78(52.3 \%)$ & $103(5.2 \%)$ & $<0.001^{\star}$ \\
\hline Headache & $23(15.4 \%)$ & $34(1.7 \%)$ & $0.017^{\star}$ \\
\hline Myalgia & $34(22.8 \%)$ & $59(3 \%)$ & $0.021^{\star}$ \\
\hline Diarrhea (n, \%) & $6(4 \%)$ & $1(0.1 \%)$ & $0.002^{\star}$ \\
\hline
\end{tabular}

Abbrveviations: ${ }^{*}$ means statistically significant. COPD: Chronic Obstructive Pulmonary Disease; DM: Diabetes Mellitus

\section{RESULTS}

This retrospective study involved 2234 patients being tested for COVID-19. The patients' symptoms, risk factors, and other demographic data are presented in Table 1. Of the patients, 1307 (58.5\%) were male and 925 (41.4\%) were female. There were 259 (11\%) PCR positive and 1975 (89\%) PCR negative patients.

In the PCR negative group, 1188 (60.1\%) patients were male and $787(39.9 \%)$ were female. The mean age was $33 \pm 11$. The number of medical staff was $282(14 \%)$. Thirty-nine (1.9\%) patients had a history of contact with people from a foreign country, including contact made when traveling abroad. In terms of clinical risk factors, 197 (10\%) patients had hypertension, 77 (3.9\%) DM, 66 (3.3\%) COPD, 39 (2\%) asthma, 35 (1.8\%) HF, and 414 (21\%) were smokers (Figure 2). On admission, 414 (21\%) patients were symptomatic and 1561 (79\%) were asymptomatic. The most common symptoms were a cough and shortness of breath.
In the PCR positive group, $121(46.7 \%)$ patients were male and $138(53.3 \%)$ were female. The mean age was 38 \pm 18.5 . Six (2.3\%) patients were medical staff. Seven (2.7\%) patients had a history of contact with people from foreign countries, including contact made when traveling abroad. In terms of clinical risk factors, $56(21.6 \%)$ patients had hypertension, 27 (10.4\%) DM, 15 (5.8\%) COPD, 16 (6.2\%) asthma, $19(7.3 \%) \mathrm{HF}$, and $74(28.6 \%)$ were smokers (Figure 2). On admission, 148 (57.1\%) of patients were symptomatic and 111 (42.9\%) were asymptomatic. Most of the symptoms reported were a cough, fever, shortness of breath, and tiredness. Other symptoms included a headache, myalgia, and diarrhea.

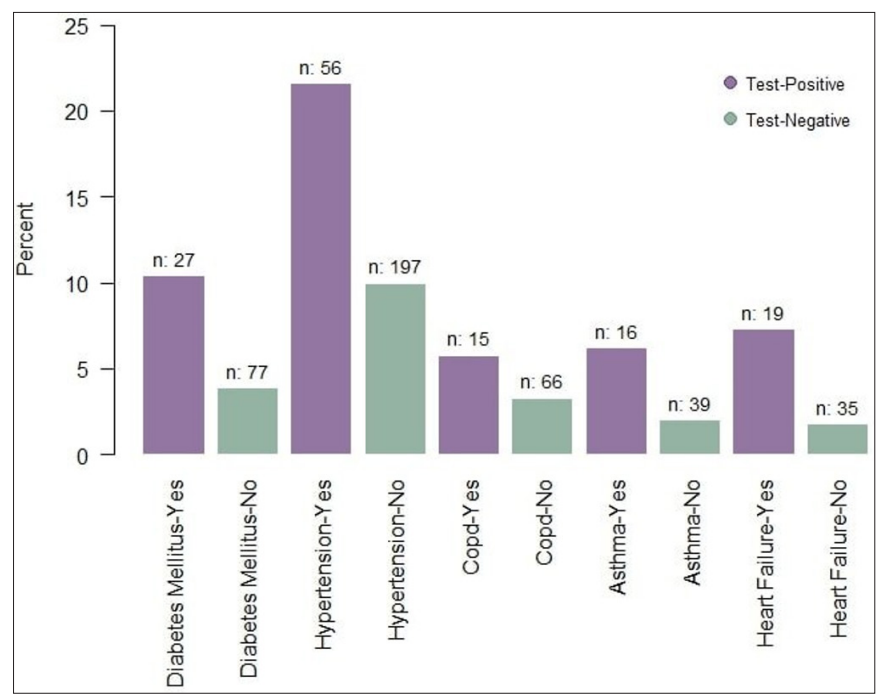

Figure 2. Graphic showing the proportion of comorbid diseases of patients

Table 2 provides information about test numbers, CT findings, treatment, and prognoses. The mean test number was 1.21 \pm 0.5 . $465 \mathrm{CT}$ scans were performed, 131 of them were PCR positive. According to the CT results, typical findings such as GGO, a crazy-paving pattern appearance, vascular dilatation in the area of GGO, air space consolidation, bronchovascular thickening in the lesion, and traction bronchiectasis were detected in 160 (7.2\%) patients. Eighty-nine of them were PCR positive. The data on CT scores for the PCR positive patients are presented in Table 3.

In accordance with their test results and clinical status, 2017 (90.3\%) patients were discharged and recommended treatment and/or isolation at home, $152(6.7 \%)$ patients needed medical treatment in hospital and $65(3 \%)$ patients needed intensive care support, including non-invasive mechanical ventilation. Of the PCR positive patients, $156(60.2 \%)$ were recommended isolation and treatment at home, $74(28.6 \%)$ were hospitalized in service during treatment, and 29 (11.2\%) needed intensive care support. Medical treatment was applied in (selected) patients according to guidelines. The antibiotics were used in 
cases of generally covered common pathogens and some atypical pathogens. When secondary bacterial infection occurred, medication was administered according to the bacterial culture and drug sensitivity results. Steroids were used in selected cases as well.

\begin{tabular}{|lcc|}
\hline \multicolumn{3}{|c|}{ Table 2. Test numbers, infection rates and prognosis of patients } \\
\hline & All (n=2234) & PCR (+) (n=259) \\
\hline Test (number, means) & $1.21 \pm 0.5$ & $2.14 \pm 0.51$ \\
CT (n, \%) & $465(20.8 \%)$ & $131(51 \%)$ \\
Infection (n, \%) & $184(8.2 \%)$ & $89(34 \%)$ \\
COVID-19 typical findings & $160(7.2 \%)$ & $89(34 \%)$ \\
Other (bacterial, fungal..) & $24(1 \%)$ & $0(0 \%)$ \\
Contamination origin (n, \%) & & \\
$\quad$ Local & $2188(97.9 \%)$ & $252(97.3 \%)$ \\
Outside & $46(2.1 \%)$ & $7(2.7 \%)$ \\
Medical staff (n, \%) & $288(12.9 \%)$ & $6(2.3 \%)$ \\
Treatment (n, \%) & $2017(90.3 \%)$ & $156(60.2 \%)$ \\
$\quad$ Home & $152(6.7 \%)$ & $74(28.6 \%)$ \\
Service & $65(3 \%)$ & $29(11.2 \%)$ \\
$\quad$ Intensive care & & \\
Prognosis (n, \%) & $2222(99.5 \%)$ & $247(95.4 \%)$ \\
$\quad$ Cured & $12(0.5 \%)$ & $12(4.6 \%)$ \\
$\quad$ Death & \\
\hline Abbreviations: CT: Computed tomography & \\
\hline
\end{tabular}

\begin{tabular}{|lc|}
\hline Tablo 3. Imaging results of patients with COVID-19 pneumonia \\
\hline COVID-19 positive (n=259) & CT (scores, mean) \\
\hline Age group ( $<6$ ) & - \\
Age group ( 6-20 ) & $1 \pm 0$ \\
Age group ( $21-50)$ & $2.71 \pm 0.19$ \\
Age group ( $>50)$ & $3.72 \pm 0.19$ \\
Sex-female & $3.31 \pm 0.21$ \\
Sex-male & $3.03 \pm 0.19$ \\
Hypertension & $4 \pm 0.19$ \\
Diabetes mellitus & $3.95 \pm 0.31$ \\
Smoking & $3.04 \pm 0.19$ \\
COPD & $3.85 \pm 0.37$ \\
Asthma & $3.91 \pm 0.39$ \\
Heart failure & $3.59 \pm 0.39$ \\
\hline Abbreviations: ${ }^{*}$ means statistically significant. COPD: Chronic obstructive \\
pulmonary disease
\end{tabular}

Of the PCR positive patients, two of them died due to COVID-19-related myocardial infarction and ten of them died due to severe pneumonia, ARDS, multiorgan dysfunction, or septic shock.

According to our study, age, sex, hypertension, diabetes, asthma, and heart failure are all associated with COVID-19 infection. It was found that females have more of an infection risk than males (OR: 1.636; $\mathrm{p}<0.001)$. Similarly, patients with hypertension (OR: $2.281 ; \mathrm{p}<0.001)$, diabetes (OR: 1.013; $\mathrm{p}=0.039$ ), asthma (OR: 2.786; $\mathrm{p}=0.001$ ), or heart failure (OR: 2.610; $\mathrm{p}=0.006$ ) have a higher risk of COVID-19 infection. It was also found that COVID-19 infection risk is significantly associated with age (OR: 0.991; $\mathrm{p}=0.039$ ) (Table 4).

\begin{tabular}{|lccc|}
\hline \multicolumn{4}{|l|}{ Table 4. Risk factors associated with COVID-19 infection } \\
\hline & $\begin{array}{l}\boldsymbol{\beta} \text { estimates with } \\
\text { standard errors }\end{array}$ & OR & p value \\
Constant $\left(\beta_{0}\right)$ & $-2.154 \pm 0.172$ & - & $<0.001^{*}$ \\
Age $\left(\beta_{1}\right)$ & $-0.009 \pm 0.004$ & 0.991 & $0.039^{*}$ \\
Sex $\left(\beta_{2}\right)$ & $0.492 \pm 0.136$ & 1.636 & $<0.001^{*}$ \\
Hypertension $\left(\beta_{3}\right)$ & $0.825 \pm 0.237$ & 2.281 & $0.001^{*}$ \\
Diabetes $\left(\beta_{4}\right)$ & $0.558 \pm 0.006$ & 1.013 & $0.039^{*}$ \\
Asthma $\left(\beta_{5}\right)$ & $1.025 \pm 0.321$ & 2.786 & $0.001^{*}$ \\
Heart failure $\left(\beta_{6}\right)$ & $0.959 \pm 0.346$ & 2.610 & $0.006^{*}$ \\
\hline Abbreviations: ${ }^{*}$ means statistically significant. & & \\
\hline
\end{tabular}

\section{DISCUSSION}

The outbreak of COVID-19 contributed to increasing morbidity and mortality rates so was announced to be a major worldwide pandemic by the World Health Organization (11). Accordingly, recent research, in terms of understanding the mechanism of progression and transmission of COVID-19, has contributed to the development of pharmacological and nonpharmacological treatment strategies (12). The clinical manifestation of the virus in humans and the increasing number of symptomatic and asymptomatic patients each day have led to a growing concern for public health.

There are some differences between urban and rural areas that may contribute to the influence of viral transmission, diagnostics, morbidity, and mortality. Socioeconomic factors, access to healthcare, and pandemic preparedness are the main factors. In past years, the H1N1 and influenza A pandemics caused high mortality rates in rural Turkey. Now, in the U.S., there is a higher percentage of elderly in the rural population (age $>60$ ) with co-existing diseases and, along with their smoking status, that increases the risk of infection and COVID-19-related severe complications $(13,14)$.

In our study, the mean age was $38 \pm 18.5$ in the PCR positive group and $33 \pm 11$ in the PCR negative group. We found that individuals aged $20-50$ and those older than 50 years had a higher risk of COVID-19 infection $(p<0.001)$. Although the area that we researched is rural, the population tested for COVID-19 was relatively young. The following factors were considered the reasons for this: first, the COVID-19 pandemic started in China, then spread to Asia, the United States, Turkey, and other European countries, so there was not enough time to prepare for the pandemic, and second, soon after the beginning of the pandemic, individuals over the age of 65 were prohibited from going outside and contacting other individuals. The people who were actively working and in contact with other individuals were young.

In our study, there were 259 patients in the PCR positive group. The incidence of infection in women was higher than in men $(\mathrm{p}<0.001)$, but the mortality rate was higher 
in males than in females. Data from single-cell RNA sequencing (scRNA-seq) suggests that the number of ACE2-expressing pulmonary alveolar type II cells are higher in men than in women. The reduced susceptibility of females to viral infections may also be attributed to sex hormones that affect the regulation of immunity. Moreover, the expression of the androgen receptor (AR) is positively correlated with ACE2. This evidence is thought to account for why men have a markedly increased risk of developing severe cases compared to women (15).

Recent studies have shown that people with diabetes mellitus, hypertension, a smoking habit, COPD, asthma, or heart failure are associated with increased infection risk and a severe prognosis. A study confirmed that diabetes (22\%) was one of the most evident comorbidities of 32 non-survivors from a group of 52 intensive care patients with COVID-19. COPD and asthma exacerbations are the major factors that cause worsening of symptoms, often lead to increased hospitalization and a poor prognosis. In intensive care units, infusion of fluids given to maintain blood pressure in patients with $\mathrm{HF}$, as well as parenteral drug administration may cause an even higher pulmonary vascular pressure, which is already high. A pulmonary edema may accompany acute respiratory distress syndrome, which increases mortality risk (1619). In relation to this evidence, our study confirmed that patients with hypertension (OR: 2.281; $\mathrm{p}<0.001$ ), diabetes (OR: 1.013; $\mathrm{p}=0.039$ ), asthma (OR:2.786; $\mathrm{p}=0.001$ ), or heart failure (OR:2.610; $\mathrm{p}=0.006)$ have a higher risk of COVID-19 infection. But interestingly, in terms of infection risk, there was no difference between patients with COPD and those without $(\mathrm{p}=0.162)$.

Cough, fever, and shortness of breath were common symptoms in both the PCR positive and PCR negative groups. Headaches were particularly common in hypertensive patients, shortness of breath was common in patients with COPD, and diarrhea was seen only in children under six years old. Some specific symptoms, such as loss of smell and taste, were not seen in any patient.

It is cricual to screen asymptomatic carriers who are an important source of COVİD-19 infections from truly healthy populations to prevent and control spreading among individuals. A recent study showed that the presymptomatic phase and asymptomatic infections account for $47 \%$ and $6.6 \%$ of transmission (20). That is why our study included symptomatic and asymptomatic patients.

In suspected patients who are PCR negative, CT evaluation is often key in the diagnosis of infection because early recognition of disease is important for timely treatment and preventing underdiagnosing (21).
In our study, $71(3 \%)$ patients repeatedly had negative PCR test results, although they had typical symptoms and CT findings. Probably, factors such as the immature development of detection technology, low patient viral load, and improper sampling contributed to the patient's negative test results.

CT scores correlated to age and the presence of comorbid diseases. Five PCR-positive patients developed ARDS, septic shock, and followed by multiple organ failure. The CT scores of each of those patients was five. Therefore, early diagnosis and timely treatment of critical cases are crucial. COVID-19 may complicate with acute coronary syndrome and even sudden cardiac death. The patients who avoid going to hospital have a higher mortality rate due to acute coronary syndrome related complications (22). Two of COVID-19 positive patients died because of COVID-19-related sudden cardiac death. There was no evidence of drug use, co-morbid conditions, or family history of the disease. Both patients were smokers.

The case fatality rate of COVID-19 is significantly associated with age and comorbidities, across the world (23). According to a report, the overall CFR in Turkey was estimated to be $1.85 \%$, but in our study population, the CFR was $4.6 \%$ (24). The probable reasons for the high mortality rate were socioeconomic factors, lack of adequate knowledge about COVID-19, a high percentage of smokers within the population, and co morbid disease. It should also be kept in mind that in addition to transmission within the population during the active epidemic period, infected patients who came to visit from outside areas also contributed to the increase in infections. In conclusion, considering the age stratification, preexisting co-morbidities, relatively limited healthcare access and resources, socioeconomic status as factors, the rural population may be at risk of higher mortality. Since there is no specific antiviral drug, measures such as frequent hand washing, wearing face masks, encouraging people to social distance, and restrictions on public gatherings and non-essential travel seem to be useful in decreasing the transmission rate. Important gaps in our knowledge of the origin, epidemiology, transmission dynamics, and clinical spectrum of the disease needed to be filled by further studies.

\section{Limitations of the Study}

The first limitation is that the study population could be larger; however, the integration of multiple centers is sometimes difficult to achieve, so we included just two centers. Second, more detailed data on the clinical course of the patients and blood tests could be given. However, the data in this study provides an early assessment of the epidemiological and clinical characteristics of people tested for COVID-19 in Adiyaman, Turkey. Another 
limitation of our study is that the medical treatment given to the patients included in our study was different from the current treatment guidelines (hydroxychloroquine and oseltamivir are no longer used in therapy).As we gain more information about COVID-19 and the number of cases continues to increase, further studies will be needed.

\section{ETHICAL DECLARATIONS}

Ethics Committee Approval: This study was approved by Clinical Researchs Ethics Committee of Adiyaman University (Date 21.07.2020, Decision No: 2020/7-32).

Informed Consent: Because the study was designed retrospectively, no written informed consent form was obtained from patients.

Referee Evaluation Process: Externally peer-reviewed.

Conflict of Interest Statement: The authors have no conflicts of interest to declare.

Financial Disclosure: The authors declared that this study has received no financial support.

Author Contributions: All of the authors declare that they have all participated in the design, execution, and analysis of the paper, and that they have approved the final version.

\section{REFERENCES}

1. Huang C, Wang Y, Li X, et al. Clinical features of patients infected with 2019 novel coronavirus in Wuhan, China. lancet 2020; 395: 497-506.

2. Chan JF-W, Yuan S, Kok K-H, et al. A familial cluster of pneumonia associated with the 2019 novel coronavirus indicating person-to-person transmission: a study of a family cluster. Lancet 2020; 395: 514-23.

3. Wang C, Horby PW, Hayden FG, Gao GF. A novel coronavirus outbreak of global health concern. Lancet 2020; 395: 470-3.

4. Li Q, Guan X, Wu P, et al. Early transmission dynamics in Wuhan, China, of novel coronavirus-infected pneumonia. N Engl J Med 2020.

5. Chen N, Zhou M, Dong X, et al. Epidemiological and clinical characteristics of 99 cases of 2019 novel coronavirus pneumonia in Wuhan, China: a descriptive study. Lancet 2020; 395: 507-13.

6. Wei X, Xiao Y-T, Wang J, et al. Sex differences in severity and mortality among patients with COVID-19: evidence from pooled literature analysis and insights from integrated bioinformatic analysis. arXiv 200313547. 2020.

7. Zheng Z, Peng F, Xu B, et al. Risk factors of critical \& mortal COVID-19 cases: A systematic literature review and metaanalysis. J Infect 2020; 81: e16-e25.

8. Sohrabi C, Alsafi Z, O’Neill N, et al. World Health Organization declares global emergency: A review of the 2019 novel coronavirus (COVID-19). Int J Surg 2020; 76: 71-6.

9. Jeyanathan M, Afkhami S, Smaill F, Miller MS, Lichty BD, Xing Z. Immunological considerations for COVID-19 vaccine strategies. Nat Rev Immunol 2020; 20: 615-32.

10. Garcia-Perez MA, Nunez-Anton V. Cellwise residual analysis in two-way contingency tables. Educ Psychol Meas 2003; 63: 825-39.

11. Cucinotta D, Vanelli M. WHO declares COVID-19 a pandemic. Acta Biomed Ateneo Parmense 2020; 91: 157-60.
12. Fehr AR, Perlman S. Coronaviruses: an overview of their replication and pathogenesis. Coronaviruses: Springer; 2015. p. 1-23.

13. Cosby AG, McDoom-Echebiri MM, James W, Khandekar H, Brown W, Hanna HL. Growth and persistence of place-based mortality in the United States: the rural mortality penalty. Am J Public Health 2019; 109: 155-62.

14. Kirakli C, Tatar D, Cimen P, et al. Survival from severe pandemic H1N1 in urban and rural Turkey: a case series. Respir care 2011; 56: 790-5.

15. Sharma G, Volgman AS, Michos ED. Sex differences in mortality from COVID-19 pandemic: are men vulnerable and women protected?. JACC Case Rep 2020; 2: 1407-10.

16. Fang L, Karakiulakis G, Roth M. Are patients with hypertension and diabetes mellitus at increased risk for COVID-19 infection? Lancet Resp Med 2020; 8: e21.

17.Johnston SL. Asthma and COVID-19: Is asthma a risk factor for severe outcomes?. Allergy 2020; 75: 1543-5.

18. Mehra MR, Ruschitzka F. COVID-19 Illness and Heart Failure: A Missing Link?. JACC Heart Fail 2020; 8: 512-4.

19. Alqahtani JS, Oyelade T, Aldhahir AM, et al. Prevalence, severity and mortality associated with COPD and smoking in patients with COVID-19: a rapid systematic review and meta-analysis. PLoS one. 2020; 15: e0233147.

20. Moghadas SM, Fitzpatrick MC, Sah P, et al. The implications of silent transmission for the control of COVID-19 outbreaks. Proc Natl Acad Sci 2020; 117: 17513-5.

21.Bernheim A, Mei X, Huang M, et al. Chest CT findings in coronavirus disease-19 (COVID-19): relationship to duration of infection. Radiology 2020: 200463.

22. Rodriguez-Leor O, Cid-Alvarez B. ST-Segment Elevation Myocardial Infarction Care During COVID-19: Losing Sight of the Forest for the Trees. JACC Case Rep 2020; 2: 1625-7.

23. Khafaie MA, Rahim F. Cross-country comparison of case fatality rates of COVID-19/SARS-COV-2. Osong Public Health Res Perspect 2020; 11: 74 .

24.Öztoprak F, Javed A. Case fatality rate estimation of COVID-19 for European countries: Turkey's current scenario amidst a global pandemic; comparison of outbreaks with European countries. EJMO 2020; 4: 149-59 